\title{
Low-Voltage-Driven Large-Amplitude Soft Actuators Based on Phase Transition
}

\author{
AU1 Ragesh Chellattoan, Arief Yudhanto, and Gilles Lubineau
}

\begin{abstract}
Soft actuators producing large motion in a short time are mostly based on stretchable polymers actuated by pneumatic pressure; they consist of bulky components, including a motor, pump/compressor, tubes, and valves. In this study, we develop a fast-responding large-amplitude soft actuator, based on a liquid-gas phase transition, which produces a compact system. The required pressure is generated solely by the electrically induced phase transition of a fluid in a cavity, mimicking the thigmonastic movements found in plants. We discuss the critical design variables to improve the performance and propose a new design for the electrodes, which are the most critical components. Our bending actuator produces large motion in $<7 \mathrm{~s}$, using a low-voltage source $(<50 \mathrm{~V})$ that allows a much faster response than the soft actuators based on phase transition currently available.
\end{abstract}

Keywords: soft robotics, phase transition, low-voltage actuation, fast response

\section{Introduction}

$\mathbf{S}$ OFT ROBOTICS IS AN emerging technology using structures made of soft and flexible materials. ${ }^{1-5}$ Recent advances in soft robots have made it possible to develop complex functionalities that mimic the various motions of living creatures such as walking, ${ }^{6}$ crawling, ${ }^{7,8}$ climbing, ${ }^{9,10}$ and gripping. ${ }^{11}$ These soft systems represent an elegant and cost-effective way to perform these important functions, compared with complex and expensive classical hard robots. The key factor to consider in the fabrication of a soft robot is the actuation mode. Current actuators use different power systems, such as pneumatic, hydraulic, ${ }^{12}$ magnetic, ${ }^{13-15}$ electric, ${ }^{16,17}$ and shape memory polymers. ${ }^{18-21}$

Existing actuation technologies have some intrinsic limitations. For example, shape memory polymers can only generate low strain (usually $<10 \%)^{18,21}$; dielectric electroactive polymers usually require a large driving voltage $(\text { several } \mathrm{kV})^{16,17}$; magnetic actuators need motors and a large set of machinery. ${ }^{13}$ Fluidic actuators (based on pneumatic or hydraulic energy) are considered to be the most promising, with respect to producing a large motion in a short time. Yet, current fluidic actuators still require pressure-generation systems that are bulky and difficult to miniaturize. ${ }^{1}$ Moreover, they are challenging to design as they typically require multiple valves or flow-controlling elements for pressurizing the chambers. An ideal actuator is compact in size, simple to control, low-cost, and able to generate a large motion or transformation in a limited time.

We believe that the ability to build fluid pressure within a soft body, using an inexpensive thermofluidic system based on liquid-vapor phase transition may be used to eliminate the need for bulky motors and compressors in classical fluidic actuation systems. By directly triggering the phase transition utilizing low-voltage equipment, such an actuation system can be made very compact. However, the technology for soft actuators based on electrically induced liquid-vapor transition is still in its infancy; certainly, it has not been optimized yet for its application to high-performance systems.

There are several existing proofs of concept of actuation based on liquid-vapor phase transition. ${ }^{15,22-25}$ For most of them, the energy chain is different, making them bulky and not portable; then, it becomes comparable with the classical fluidic actuation system. For instance, Boyvat et al. ${ }^{15}$ relies on additional bulky magnetic equipment. In contrast, some studies utilized an external thermal or photoactivated phase transition, which is not operated using direct electric power and often limited to small deformation of soft body. ${ }^{22,24,25}$ Although there are few studies reported on electrically driven thermofluidic actuators, either they are limited to 
small membrane expansions or slow responding artificial muscles with small deformation due to inefficient planar heating system. ${ }^{23,26-28}$

As of today, to the best of our knowledge, no research that focuses on identifying the key parameters (properties of heating element and fluid, the structural advantage of soft body), guiding the performance of such thermofluidic system (significant motion and fast response) has been done to design an electrically driven, compact, and better-performing phase change actuator.

\section{Objective}

In this study, we propose a new thermofluidic actuation system producing a large motion in a short time operated using electric power. We are inspired from the electrically induced pressure change in the chambers of the "pulvinus" of some plants such as those from the bean family, for example. In these plants, the bending is generated by the deformation of the pulvinus through a quick pressure change. ${ }^{29,30}$ This pressure change is directly triggered by electrical signals making the actuation system very compact. In our approach, a cavity is designed to incorporate the adequate amount of a selected fluid to build the required pressure of actuation when vaporized. The progressive change of this liquid to vapor, produced by Joule heating, ensures that the deformation is sufficient through a large volumetric expansion. In such a system, sufficient pressure can be generated within the soft body using a low-voltage source, and so, the whole system can remain compact.

To improve the time response of this new actuator, we pay special attention to the design of the heating element. We introduce a rigorously selected porous electrode with adequate electrical properties such that the generation of heat is optimized. This is expected to improve the speed of actuation in the forward direction, which is based on the active heating of the fluid. The reverse motion, however, remains an issue, as the passive heat dissipation is often not as fast as the heating. To improve the reverse-motion speed, we adopt a structure with a corrugated shape, thin membrane walls, and a large surface area that facilitates a better dissipation of heat to the environment.

Finally, we demonstrate that this technology can be used to design low-voltage-driven robotic grips that are fastresponding, self-sustained, powered with a simple electrical connection, and able to execute basic functionality (gripping). This clearly paves a way to the production of fastresponding modular robotic systems by simplifying drastically the connections, compared with pneumatic-based systems. It also allows miniaturization, allowing a weight and sizefootprint reduction of the actuator.

\section{Materials and Methods}

\section{Working principle}

Our fast-responding compact actuation system is inspired by the quick defensive bending (thigmonastic) movement in a

F1 well-known plant named "Mimosa Pudica" 31,32 (Fig. 1a-c). The thigmonastic movement of the petiole in this plant is generated by an electrochemical three-step reaction: (1) input stimulus by touch; (2) transmission of electrical signal to the cell walls of specialized motor organs, called the pulvini; and
(3) biochemical, hydrodynamical, and mechanical responses in the cells. The electrical stimuli induce a rapid change in hydraulic pressure (turgor pressure) in small cavities (within the cells) of the pulvinus through water transport produced by changes in ion concentrations (Fig. 1b). The volume change in the cell cavities of the pulvinus is the basis of the quick bending movements.

Inspired by the role of the electrical signal to induce the change in turgor pressure in the cavities of the pulvinus, the working principle of the soft actuator relies on the rapid buildup of vapor pressure inside a cavity comprising an electric heating element and a liquid an electrothermal reaction. Figure 1d (left) shows the initial stage of the actuator corresponding to the reference configuration, for both the pressure and volume [both change of pressure $(\Delta P)$ and volume $(\Delta V)$ are zeros]. Most of the fluid is in its liquid phase, and no actuation is generated. When the electrical current $I$ is supplied to the heating element, heat $(Q=V I)$ is generated by Joule effect in the electrode (Fig. 1d, right). This heat is then transferred to the liquid and subsequently vaporized, resulting in the volumetric expansion of the soft body. For the enabling reverse actuation, the heat supply is stopped by cutting off the electrical current, and the heat is dissipated by passive cooling of the soft element.

\section{Liquid selection}

First, we selected the liquid, based on its total heat, which is the summation of the sensible heat, $\Delta H_{\text {sen }}$, required to bring the liquid from room temperature to the boiling point and the latent heat, $\Delta H_{v a p}$, required to change the phase from liquid to vapor. $\Delta H_{\text {vap }}$ is generally much larger than $\Delta H_{\text {sen }}$ (Fig. 1f) and is thus the main parameter to consider when choosing the liquid. It is obvious that liquid with lower latent heat, for example, dichloromethane or acetone, could generally actuate the soft body faster since less heat is needed for the phase transition.

Our second selection parameter was the boiling temperature of the liquid, $T_{b}$. Figure $1 \mathrm{e}$ shows the relationship between the total heat and the boiling temperature of some liquids. If the boiling point is too close to the temperature of the surrounding environment, this may result in an unwanted actuation by spontaneous vaporization of the liquid. Having a high boiling point is then a way to separate the functional temperature (that can then be only achieved through the heat provided by the electrode) from the environment temperature.

To span a wide range of physical characteristics, we considered two liquids: ethanol (high total heat and boiling point) and acetone (low total heat and boiling point).

\section{Selection of the heating element}

Several criteria were used to select the heating element (also referred to as "electrode"). First, the heating element should be porous with a high surface-to-volume ratio (geometrical aspect) to optimize the heat transfer to the liquid. It should be flexible and preferably have a low modulus to facilitate large deformation of the soft body (mechanical aspect). It should be able to generate sufficient heat by Joule effect under low voltage; it should also possess a good capacity to carry electric current (electrical aspect). Preferably, the heating element should have a low thermal conductivity 

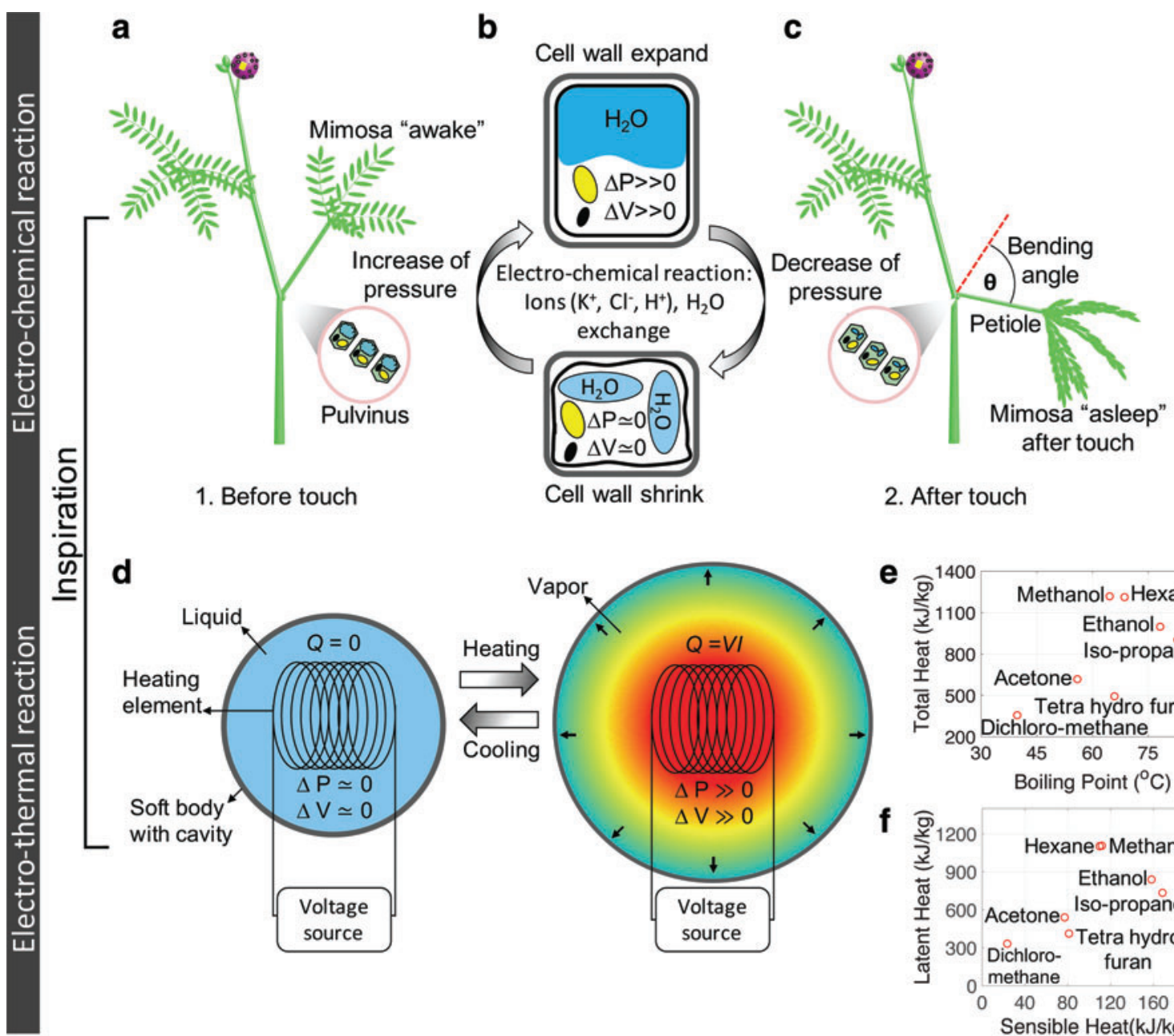

2. After touch

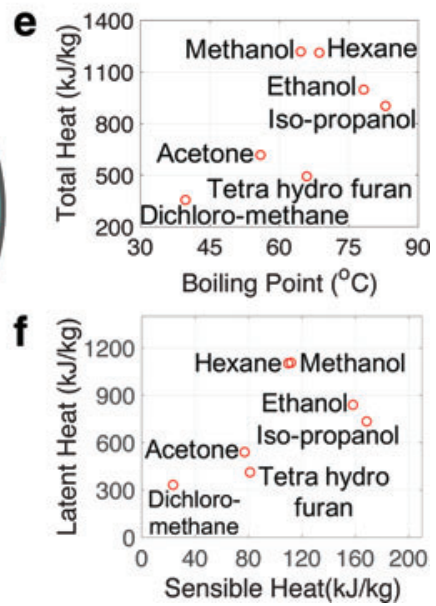

FIG. 1. (a) Turgid cells in the pulvinus of the Mimosa Pudica that keep petiole straight. (b) Schematic diagram showing the loss of turgidity, through water loss triggered by chemical potential, after touch of the leaf. (c) Defensive bending movement resulting from shrinkage of the cells. (d) Working principle of the electrically driven actuator based on liquid-tovapor phase transition. (e) Total heat versus boiling temperature at $1 \mathrm{~atm}$ pressure and (f) latent heat versus sensible heat for some common liquids. Color images are available online.

for improving the heat transfer coefficient (thermal aspect). Based on all these criteria, we selected a yarn made of microfibers of steel as the heating element.

The selection of steel wool came after a sequential optimization process, reviewed in detail as follows. We considered many options to produce the heating element in high surfaceto-volume ratio system, including (but not limited to) the use of porous metal foam, porous carbon nanotube (CNT)-polymer composite or silver nanowire (AgNW)-polymer sponge. Although the metal foam had a large surface-to-volume ratio, it was not flexible enough. The CNT-polymer composite was flexible and stretchable, but it possessed a high resistance (usually in the order of ... $\mathrm{k} \Omega$ ) because of the tunneling resistance at the interparticle junctions. Thus, a CNT-polymer composite would require a voltage that is too high for generating enough heat by Joule effect. The detrimental effect of the contact resistance in a CNT network might be overcome by using AgNWs with high aspect ratio, but the diameter of an individual nanowire, $50-100 \mathrm{~nm}$, would be too small for a stable system. AgNWs would easily melt, even at low current (usually in the range of milliampere). Moreover, due to the high piezoresistivity in percolation-based nanomaterials, the resistance in both CNT- and AgNW-based conductive polymers might significantly vary, during the deformation. ${ }^{36,37}$ This is not desired for our system, as it requires a constant resistance to operate at constant input power even under large deformation.

The steel wool yarn (diameter $2 \mathrm{~mm}$ ) that we fabricated contains a random network of numerous steel microfibers with an individual diameter of $\sim 25 \mu \mathrm{m}$, that make the structure porous and flexible enough to accommodate a large deformation. It possesses a good current-carrying capacity (up to $0.25 \mathrm{~A} / \mathrm{mm}^{2}$ ), compared with AgNWs. The electrical resistance of the steel wool can be tuned by choosing both the diameter of the yarn and the fibers. An adequate resistance is essential to generate the necessary amount of heat, in a limited time, using a low-voltage equipment (usually resistance of $10-30 \Omega$ under $60 \mathrm{~V}$; see Calculation of Size of the Heating Element section in Supplementary Data for the design approach). Steel possesses a thermal conductivity lower than those found in other metals such as aluminum and brass; its heat transfer coefficient is, therefore, relatively high. ${ }^{33}$ Having a material with a low thermal conductivity makes it possible 
for the solid/fluid wall temperature to rapidly increase under heat input. This results into an activation of the potential vapor generating points, that is, an increase of the heat transfer coefficient. ${ }^{33}$ Finally, steel wool is safe to handle and very economical compared with nanomaterials. To the best of our knowledge, research studies on the use steel wool as a heating element for soft actuators has never been reported, despite its great potential for making flexible compact fast-responding thermofluidic actuation systems.

\section{Structural design of the soft actuator}

The objective of our research is to introduce a design concept that can be used for many types of actuation mode in soft robotics such as twisting, elongation, and bending. In this study, simply for demonstration purpose, we chose a structure with the ability to undergo bending deformation, a mode that is widely applicable to various functionalities such as walking, climbing, and gripping. We used a design similar to the design recently introduced for bending, ${ }^{38}$ but powered by pneumatic systems, instead of thermofluidic system.

Figure 2a shows the components of the soft actuator con- $4 \mathrm{~F} 2$ sisting of a top corrugated enclosure with chambers (silicon rubber, EcoFlex-50), a heating element (steel wool) that is connected to copper wires (source of electrical power), a cotton fabric layer that introduces the stiffness asymmetry needed for the bending, and a bottom flat enclosure (EcoFlex-50). Figure $2 b$ and $c$ shows the schematic assembly of the soft actuator and the actuator filled with liquid (ethanol). The distribution of heating element within the soft body is shown

\section{a Components}

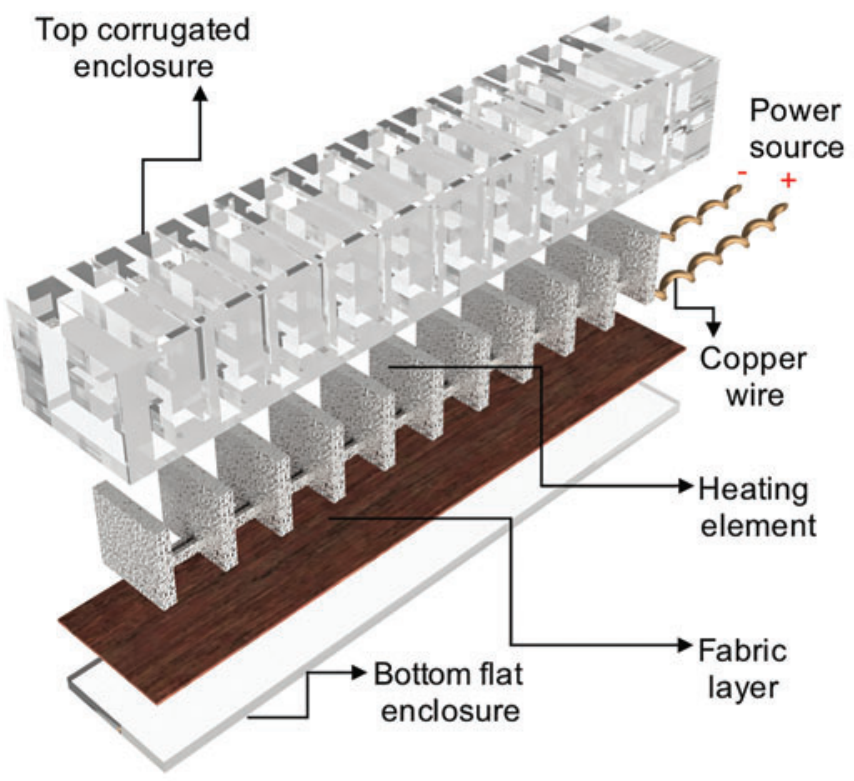

\section{b Assembly}
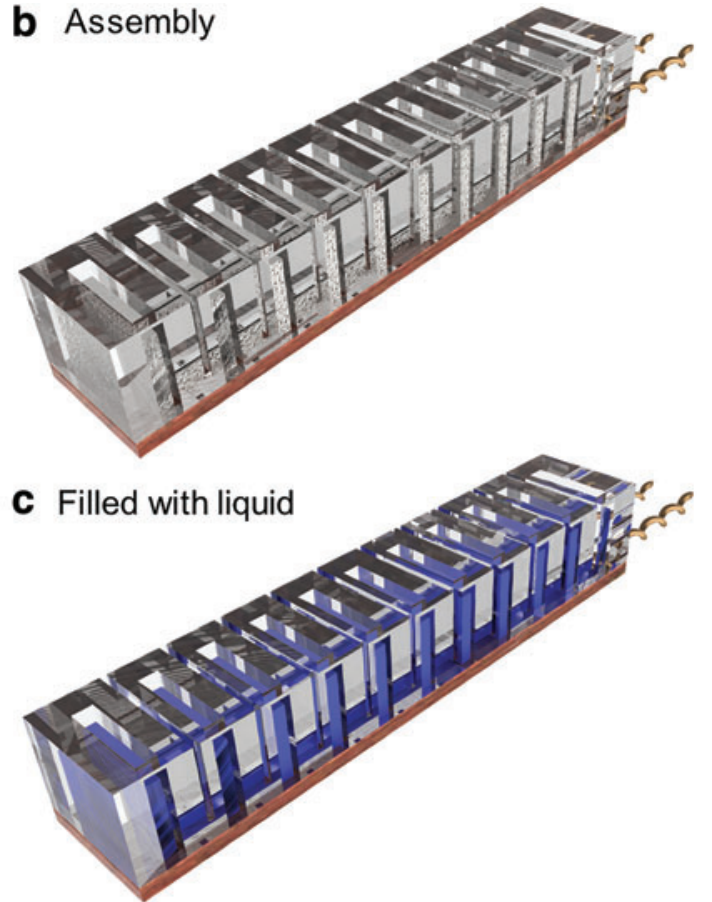
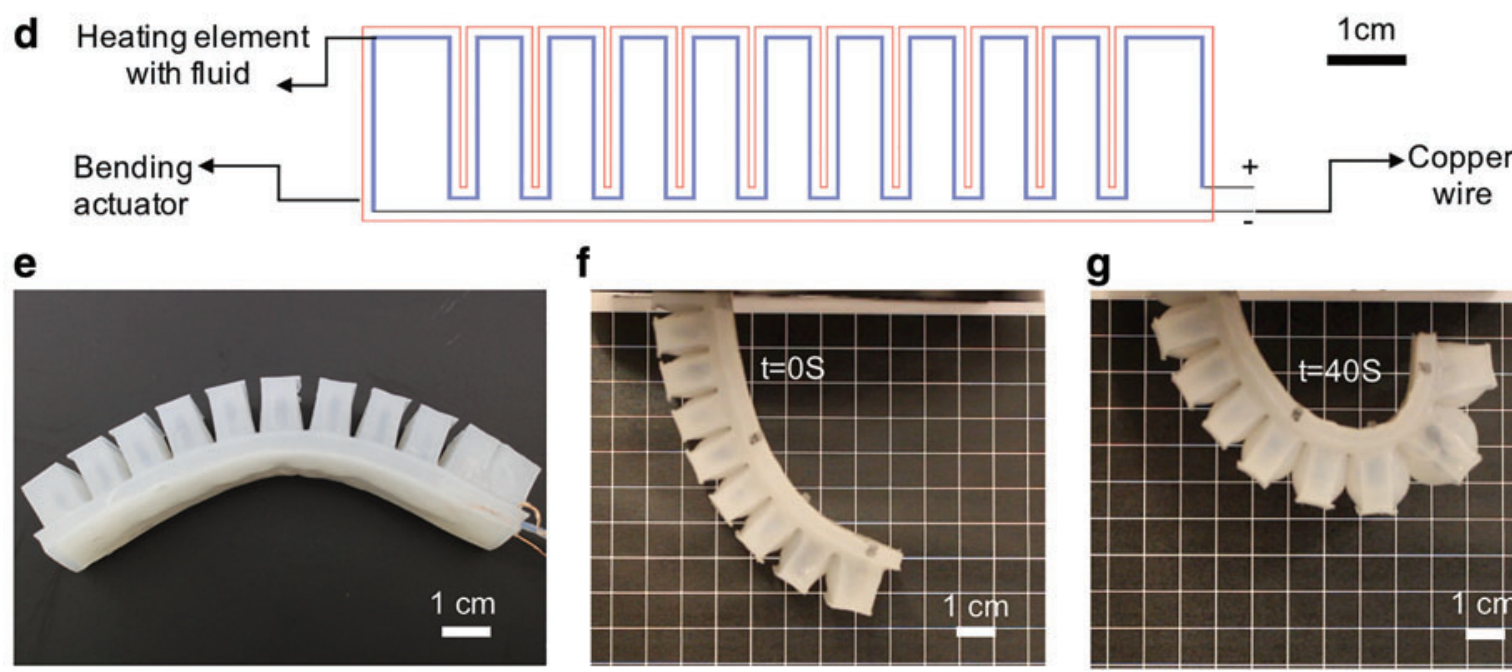

g

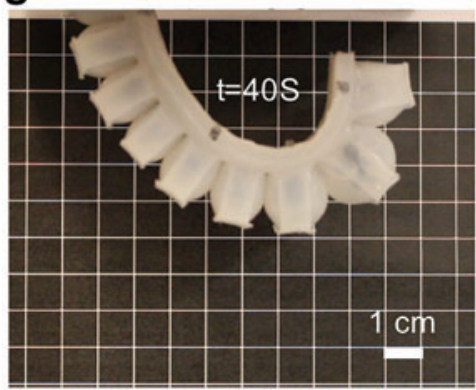

FIG. 2. (a) Components of the soft actuator, (b) assembly of the soft actuator, (c) actuator filled with liquid, (d) a 2D schematic diagram showing the distribution of heating element, (e) actual soft actuator, (f) undeformed, and (g) deformed actuators (input power: $30 \mathrm{~W}$ ). Color images are available online. 
in the two-dimensional schematic diagram (Fig. 2d). Figure 2e shows the actual soft actuator that has been fabricated. Injecting the current to the steel wool caused the liquid to vaporize, and created an internal pressure within the chamber. Owing to its unsymmetrical structure and bottom stiff layer, the actuator would deform through bending (Fig. 2f, g). We selected a corrugated chamber-like structure because of the reduced thermal resistance resulting from its large surface area. This can improve the heat transfer in passive cooling and improve the speed of reverse actuation (see Discussion on Enhancement of Passive Cooling section in Supplementary Data for details).

Although the actuator used in this study is molded, it can also be three-dimensional (3D)-printed very easily and fully electrically powered, making it a very simple technology.

\section{Results and discussion}

\section{Characterization of the heating element}

Resistance. We first measured the resistance of five different grades of steel wool (Grade-0, 00, 000, 0000,
00000) yarns. We found that the diameter of a single microfiber gradually decreased from Grade- $0(\approx 100 \mu \mathrm{m})$ to Grade-00000 $(\approx 25 \mu \mathrm{m})$, as shown in Figure 3a. Figure $3 \mathrm{~b}<\mathrm{F} 3$ shows that the resistance $R$ of the Grade-00000 yarn is consequently higher than that of Grade- 0 due to its smaller diameter. From this measurement, we selected the Grade00000 steel wool for fabricating the heating element. To test its performance, we prepared a sample yarn, by twisting and compacting the Grade-00000 steel wool to obtain a $100 \mathrm{~mm}$ length (L) and a $2 \mathrm{~mm}$ diameter (d) (Supplementary $<$ AU3 $<$ SF2 Fig. S2b). The scanning electron microscope (SEM) image shown in Figure 3c shows that Grade-00000 retains its porous structure, even after the compaction.

Resistance under mechanical loading. We measured the resistance of the Grade-00000 steel wool yarns, placed under mechanical loading (compression and bending). Details are presented in the Experimental Section. Results showed that a compressive extension of $1.5 \mathrm{~mm}$ along the diameter of the yarn (equivalent to $75 \%$ compressive strain) changed the resistance from 4.8 to $4.0 \Omega$, merely a $16 \%$ change that is a
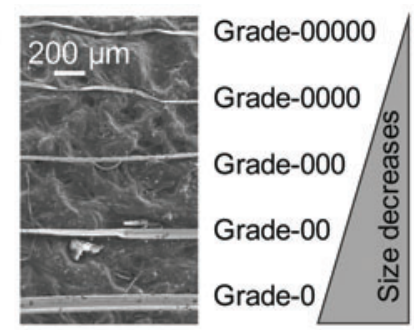

d

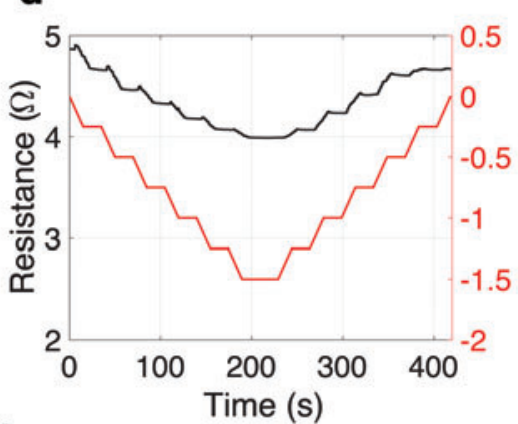

g

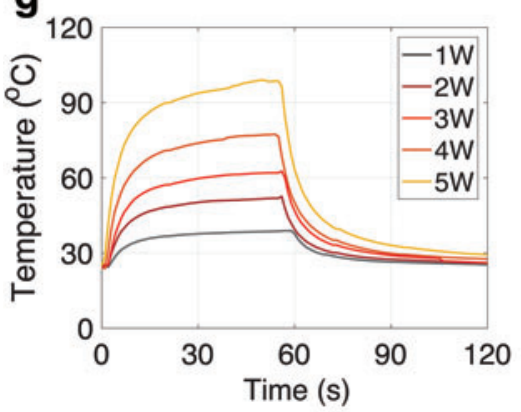

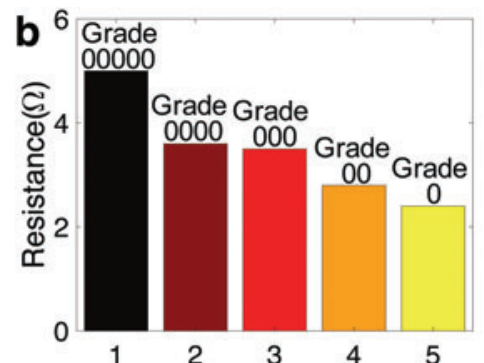

e

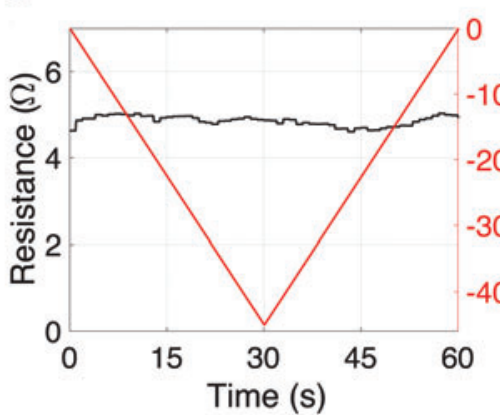

h

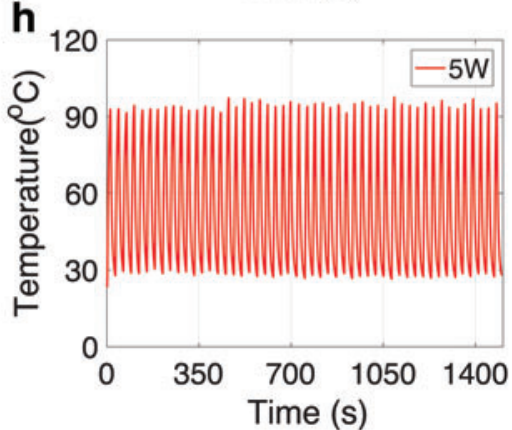

C

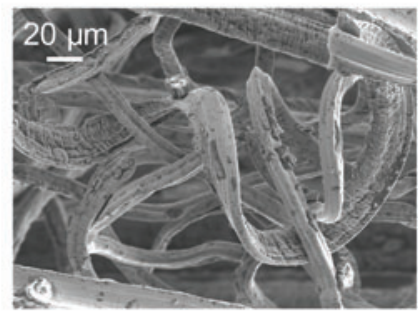

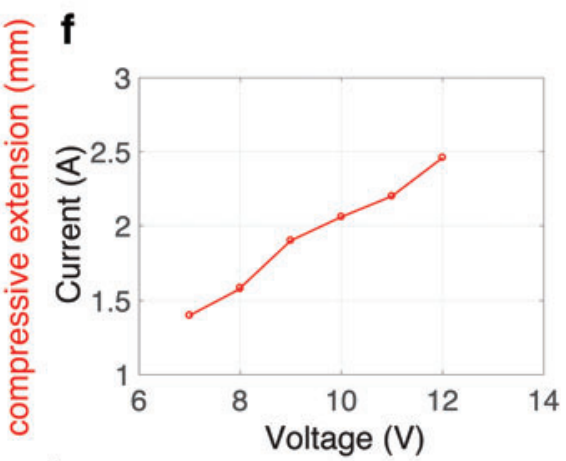

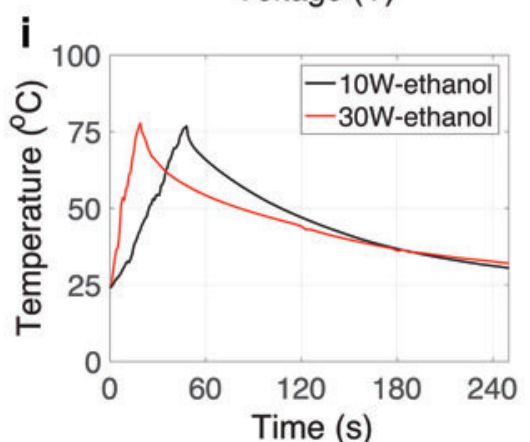

FIG. 3. (a) SEM image of a single microfiber of steel wool of various grades, (b) resistance of the various grades of a steel wool yarn, (c) SEM image of the porous structure in Grade-00000, (d) resistance change in Grade-00000 yarn under compression, (e) resistance change in Grade-00000 yarn under bending, (f) current stability in Grade-00000 yarn, (g) temperature change in Grade-00000 yarn under different power inputs, (h) cyclic heating-cooling performance of Grade00000 yarn, and (i) temperature of Grade-00000 yarn immersed in ethanol under different power inputs. SEM, scanning electron microscope. Color images are available online. 
unlikely to affect the current stability (Fig. 3d). A bending load of up to $45 \mathrm{~mm}$ compressive extension (equivalent to a bending deformation of $>180^{\circ}$ angle) did not modify the resistance of the yarns either (Fig. 3e). These results led us to conclude that the mechanical loading does not modify the resistance of yarns made of Grade-00000 steel wool and, therefore, guarantees that the performance of the system does not vary significantly during geometrical transformations.

Current stability. The linear relationship between the applied voltage and the measured current (ranged between 1.20 and 2.46 A), shown in Figure 3f, suggests that the steel wool yarn (Grade-00000) is a stable ohmic conductor under Joule heating.

Thermal stability. We applied an electrical current to Grade-00000 yarns with various input powers (from 1 to 5 $\mathrm{W}$ ), and measured their increases in temperature, in open air (a thermal image is shown in Supplementary Fig. S2c). Figure $3 \mathrm{~g}$ shows that a stable temperature is achieved after injecting a current for $1 \mathrm{~min}$. Increasing power from 1 to $5 \mathrm{~W}$ increased the average temperature from 40 to $100^{\circ} \mathrm{C}$ (sufficient to boil the selected liquids). With a $5 \mathrm{~W}$ input power, the temperature of the yarn increased from room temperature to $80^{\circ} \mathrm{C}$ within $10 \mathrm{~s}$, and so our yarn outperformed the recently reported other flexible heating element made by nanomater- ials. ${ }^{36,37}$ Cyclic heating-cooling was also performed on Grade-00000 yarn by applying a maximum input power of 5 $\mathrm{W}$ for $1500 \mathrm{~s}$. Figure $3 \mathrm{~h}$ shows that no degradation of the temperature, indicating a good heating-cooling performance. The ability to attain the boiling point of a selected liquid, ethanol, is also measured for the Grade-00000 yarn by immersing it in a glass tube containing $1.5 \mathrm{~mL}$ ethanol (Fig. $3 \mathrm{i}$ ). Figure 3 shows that the time required to reach the $T_{b}$ of $4 A U 4$ ethanol can be suitably adjusted by increasing the input power (which depends on cavity size of soft actuator and fluid quantity). For example, time decreased from 47 to $18 \mathrm{~s}$ when power increased from 10 to $30 \mathrm{~W}$.

Response time of soft actuator. The response time of a soft bending actuator filled with ethanol (see Experimental Section for fabrication details) was evaluated for different input energies (30-45 W) by capturing the deformed shape, using a video camera. The initial and deformed configurations under an input power of $30 \mathrm{~W}$ are shown in Figure $2 \mathrm{f}$ and $\mathrm{g}$. The trajectory was evaluated from the captured video, at different time intervals (Fig. 4a, b). During the forward $<\mathrm{F} 4$ actuation (Fig. 4a), the bending actuator produced a large motion for a low input power $(30 \mathrm{~W})$, in $40 \mathrm{~s}$; which can be considered to be a fast movement in such big structure (size $1 \times \mathrm{w} \times \mathrm{h}=110 \times 20 \times 20 \mathrm{~mm}$ and weight $42 \mathrm{~g}$ ). The trajectory of reverse actuation (Fig. 4b) showed the finger's position
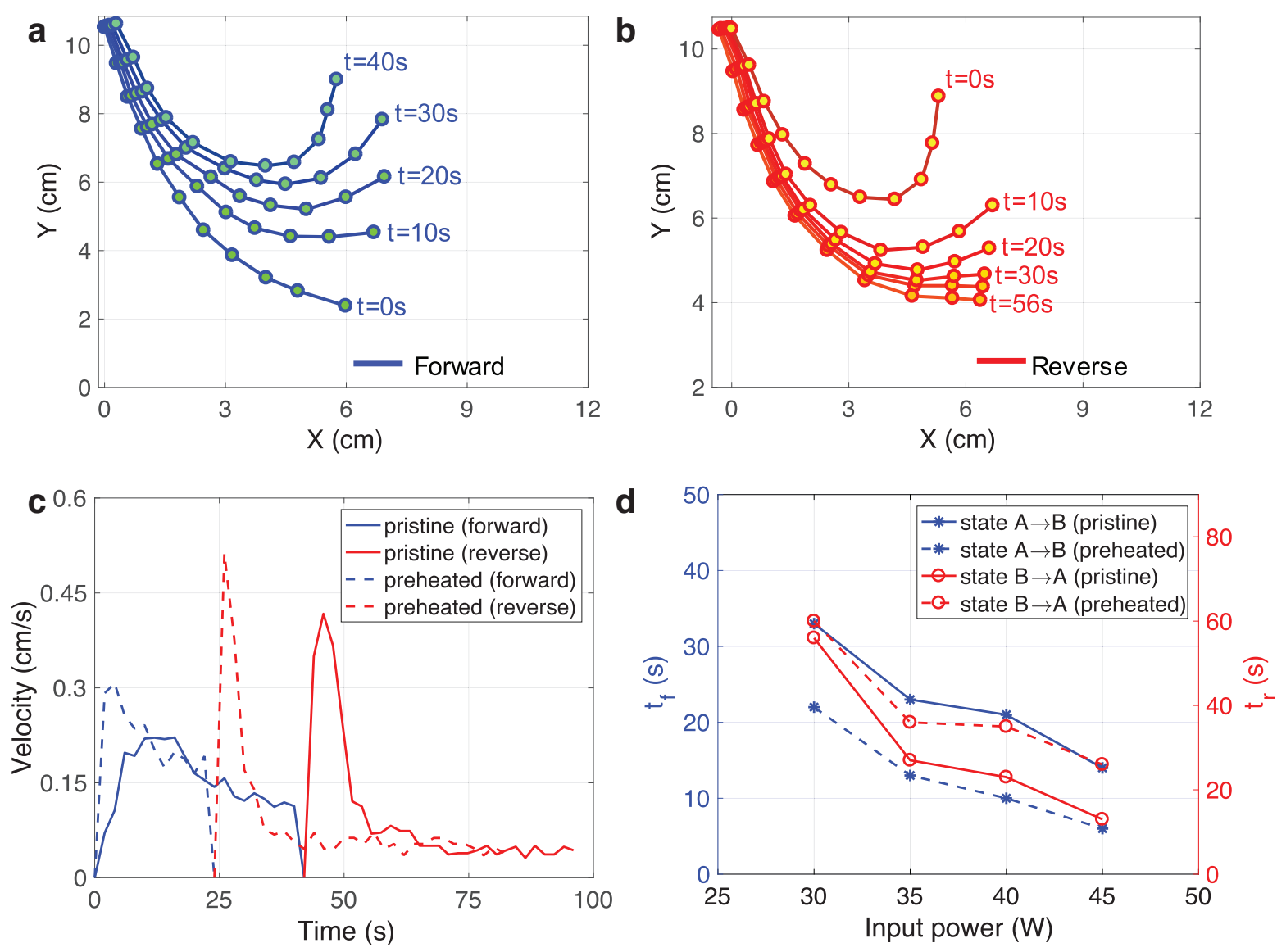

FIG. 4. Soft bending actuator using ethanol: trajectory during (a) forward and (b) reverse motion (input power of $30 \mathrm{~W}$ ), (c) velocity at different time intervals, (d) change in response time during forward and reverse actuation for different power inputs. Color images are available online. 
significantly changed during the first $20 \mathrm{~s}$, and then settled down to a plateau.

We further evaluated the velocity of the tip of the actuator (Fig. 4c) to better understand the speed of actuation. To do so, we needed to separate clearly the situation corresponding to a pristine actuator (for which the actuator is cold and is going through its first cycle) and the situation of a preheated actuator (that is the typical situation during its second and following cycles). For a pristine actuator, the velocity of the forward motion almost increased linearly in the first $10 \mathrm{~s}$, and then stabilized at $\sim 0.21 \mathrm{~cm} / \mathrm{s}$, and then declined slightly. The linear increase of the velocity during the first $10 \mathrm{~s}$ involved a gradual boiling of liquids, followed by a steady generation of vapor that led to a stabilization of velocity at its maximum value. The further decline in velocity is due to the shift in the motion of actuator from horizontal to vertical direction, as additional work against gravity is then required. The velocity analysis in reverse direction ( $40 \mathrm{~s}$ onward) revealed a sudden hike in the velocity in reverse direction during the first few seconds, which then progressively stabilized in time to finally decrease at a later stage. The high initial velocity came from stored gravitational energy in the forward direction. As the system relies on passive cooling method, the velocity decreased with further motion. In the subsequent cycle, in which the system is already preheated, we found that the slope of velocity graph increased sharply in the forward di- rection, and that the value of the maximum velocity also increased to $0.31 \mathrm{~cm} / \mathrm{s}(\sim 48 \%)$. The velocity of reverse actuation also increased slightly in the preheated system (from 0.42 to $0.51 \mathrm{~cm} / \mathrm{s}$, i.e., $\sim 21 \%$ ).

We define the time of actuation as the time required to change the configuration from states $A$ to $B$ as shown in Supplementary Figure S2a. Figure 4d shows the time of actuation in forward $\left(t_{f}\right)$ and reverse motions $\left(t_{r}\right)$. Figure $4 \mathrm{~d}$, clearly shows that, as we increased the input energy from 30 to $45 \mathrm{~W}$, the $t_{f}$ was gradually decreased. The time was reduced from 33 to $15 \mathrm{~s}$ and from 22 to $7 \mathrm{~s}$ for the pristine and preheated samples, respectively. For a big structure of $42 \mathrm{~g}$ experiencing large transformations, this represents a very fast response, comparable with the performance of a pneumatic actuator, and much better than for the thermofluidic system recently reported (even a small deformation took $>1 \mathrm{~min}$ ). ${ }^{28}$ Results for the reverse actuation (Fig. 4d) show a decrease in time as the power increases, for both the pristine and preheated samples. In general, we observed that although $t_{r}$ is $>20 \mathrm{~s}$ in the preheated sample, it is far better than in a study reported previously, in which it takes $>1 \mathrm{~min}^{28}$

The increase in speed of the preheated system was addressed by examining the pressure and temperature inside the cavity (Fig. 5a, b); we found that our selected heating element 4 F5 is good enough to generate the required pressure $(\sim 15 \mathrm{kPa})$ in a limited time (Fig. 5a). When a pressure gauge was
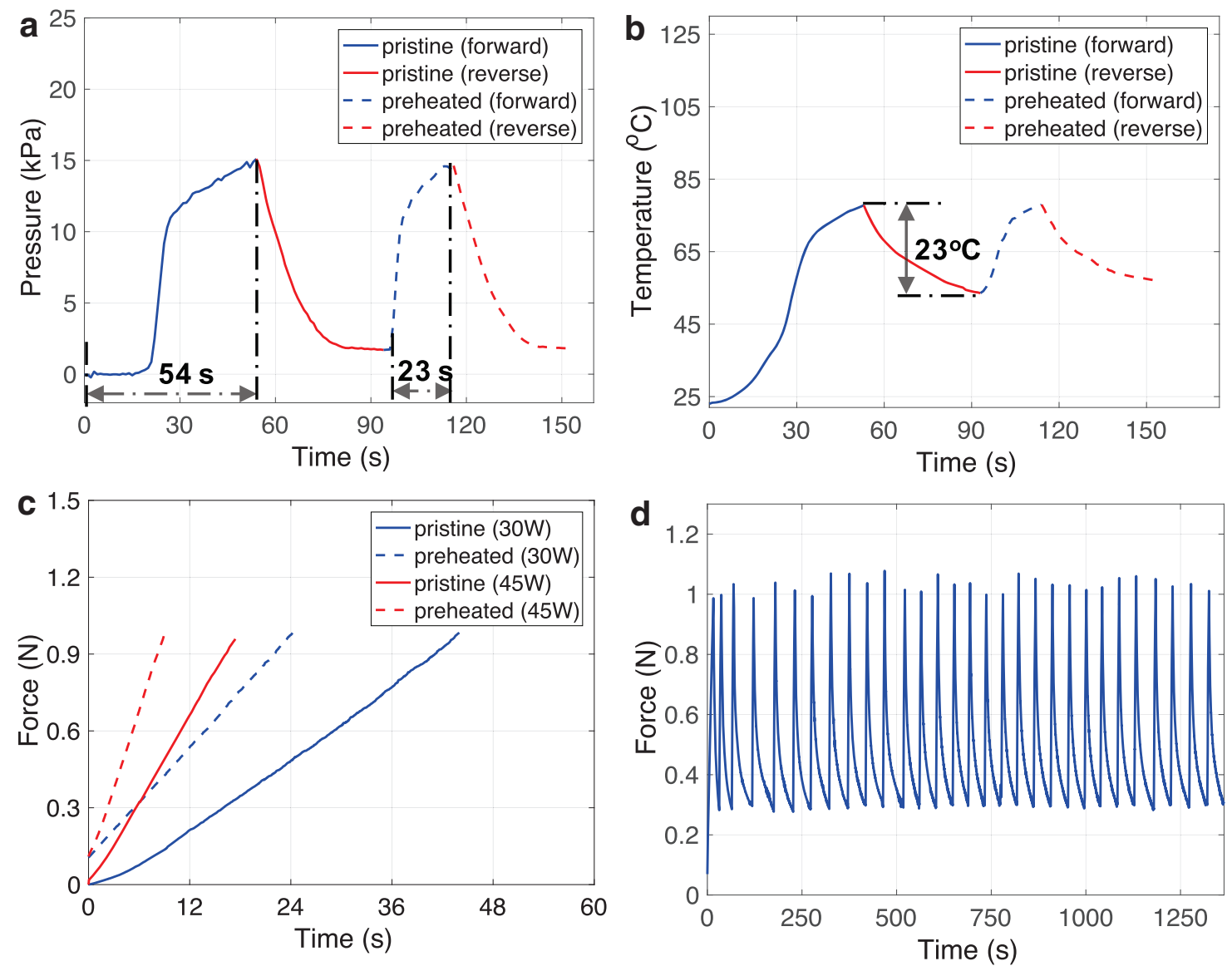

FIG. 5. Analysis of soft bending actuator using ethanol: change in (a) pressure and (b) temperature within the cavity versus time, (c) time response of generated force for different power inputs and (d) cyclic force analysis for $45 \mathrm{~W}$ power input. Color images are available online. 
connected, it took bit longer time to start the actuation process, due to the additional volume of pipe fittings. The pressure developed much faster in the preheated state $(23 \mathrm{~s})$, compared with the pristine state $(54 \mathrm{~s})$. A temperature analysis showed that the temperature inside the cavity of pristine sample increased to $77^{\circ} \mathrm{C}$ during the forward actuation (Fig. 5b), and dropped to only $54^{\circ} \mathrm{C}$ before starting the next cycle. This means that part of the supplied energy was stored in the system as internal energy and could act as a thermal reservoir to supply the energy for the next cycle of actuation; this explains why the velocity in forward actuation is elevated in a preheated system.

Figure $5 \mathrm{c}$ shows the amount of force generated by the tip of the bending actuator for a given time. Under $30 \mathrm{~W}$, the pristine sample could generate a $1 \mathrm{~N}$ force in $44 \mathrm{~s}$, but with the preheated system it took only $\sim 27 \mathrm{~s}$ to generate the same force. As power increased to $45 \mathrm{~W}$, the system generated the same amount of force in a very limited time, $\sim 17$ and $9 \mathrm{~s}$ for the pristine and preheated systems, respectively. The repeatability of our system is demonstrated in Figure 5d, by performing a cyclic force analysis under $45 \mathrm{~W}$ energy input.

Thermal analysis and tunable temperature actuation. The working temperature of the sample, which is crucial for operating safely, depends on the boiling point of the working liquid and the thermal resistance of the wall.

Figure 6 shows thermal images of a pristine sample operated at $30 \mathrm{~W}$, with ethanol as a working fluid. We found that the temperatures inside the cavity (illustrated in Fig. 5b) and on the thin chamber walls (Fig. 6) were much higher $\left(\sim 77^{\circ} \mathrm{C}\right)$ than the temperature on the thick gripping surface $\left(50^{\circ} \mathrm{C}\right)$, as shown in Figure 6.

Some important points need to be noted with respect to the cooling of our soft bending actuator. First, the low thermal resistance resulting from the small thickness of the side walls of each chamber improves the conduction heat transfer. At the same time, the high thermal resistance of the bottom layer keeps the outer gripping surface at its lowest possible temperature $\left(50^{\circ} \mathrm{C}\right)$ for a safe operation, which can be further tuned by increasing the thickness. In addition, the large expansion of the chamber walls (bulged state) during the actuation reduces the thickness and thermal resistance even more, which can also further improve the heat transfer (see
Discussion on Enhancement of Passive Cooling section in Supplementary Data; Supplementary Fig. S1a-c). The sec- $\varangle$ SF1 ond aspect to underline is the fact that the corrugated design with 11 chambers of the top layer can significantly increase the surface area, in comparison with a simple rectangular shape (Supplementary Fig. S1d). Such increase in area improves the convection heat transfer. Finally, it should be noted that an active cooling system, such as a Peltier- or a fluid-based cooling, may improve the time required for reversibility; however, it requires additional energy and space.

Reducing the total heat and boiling point of the liquid appeared to reduce both the time of actuation and the operating temperature. Therefore, we also evaluated the time of actuation using acetone, a liquid with a much lower total heat (38\%) and boiling point (28\%) than those of ethanol. Results are shown in Figure 7a. For a given lower input power, the $4 \mathrm{~F} 7$ acetone showed a significantly faster response than the ethanol. But for higher power inputs, the difference in time of actuation between ethanol and acetone for both $t_{f}$ and $t_{r}$ decreased gradually (Figs. 4d and 7a).

An analysis of the pressure development under $30 \mathrm{~W}$ (Fig. 7b) showed that the pressure inside the channel increased considerably faster with acetone $(32 \mathrm{~s})$ than with the ethanol, even faster $(13 \mathrm{~s})$ with a preheated condition. The temperature curve (Fig. 7c) shows that the temperature increased to $54^{\circ} \mathrm{C}$ during actuation, and then decreased to $42^{\circ} \mathrm{C}$ after cooling, a difference of only $12^{\circ} \mathrm{C}$. This difference was much higher with the ethanol $\left(\sim 23^{\circ} \mathrm{C}\right)$. That is one of the reasons why in acetone the reverse actuation is a little faster, especially with a lower input regime. We found that the time required to generate $1 \mathrm{~N}$ force was much lower for the acetone than for the ethanol in both pristine and preheated conditions, even at low power of $30 \mathrm{~W}$ (Fig. 7d).

These results led us to conclude that to improve the speed of actuation, choosing a liquid with a low total heat is the best option. However, if such liquid has a low boiling point, the actuator will be sensitive to changes in the environmental temperature and will have to operate at a temperature below the boiling point. If the change in environmental temperature is a major concern, it is then preferable to use the liquid with a higher boiling point. It is worth noting that, although acetone is used in day-to-day applications such as polish removers, severe leaks could lead to safety issues.
FIG. 6. Thermal image of the soft actuator during forward and reverse motions. Color images are available online.

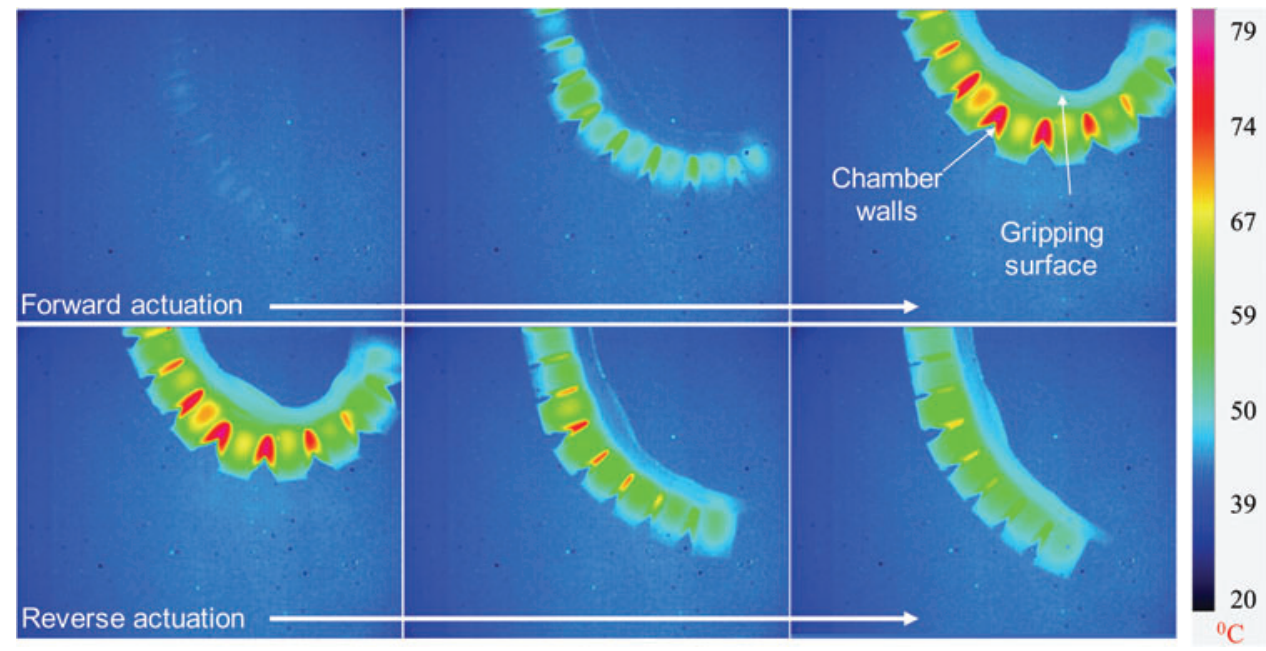



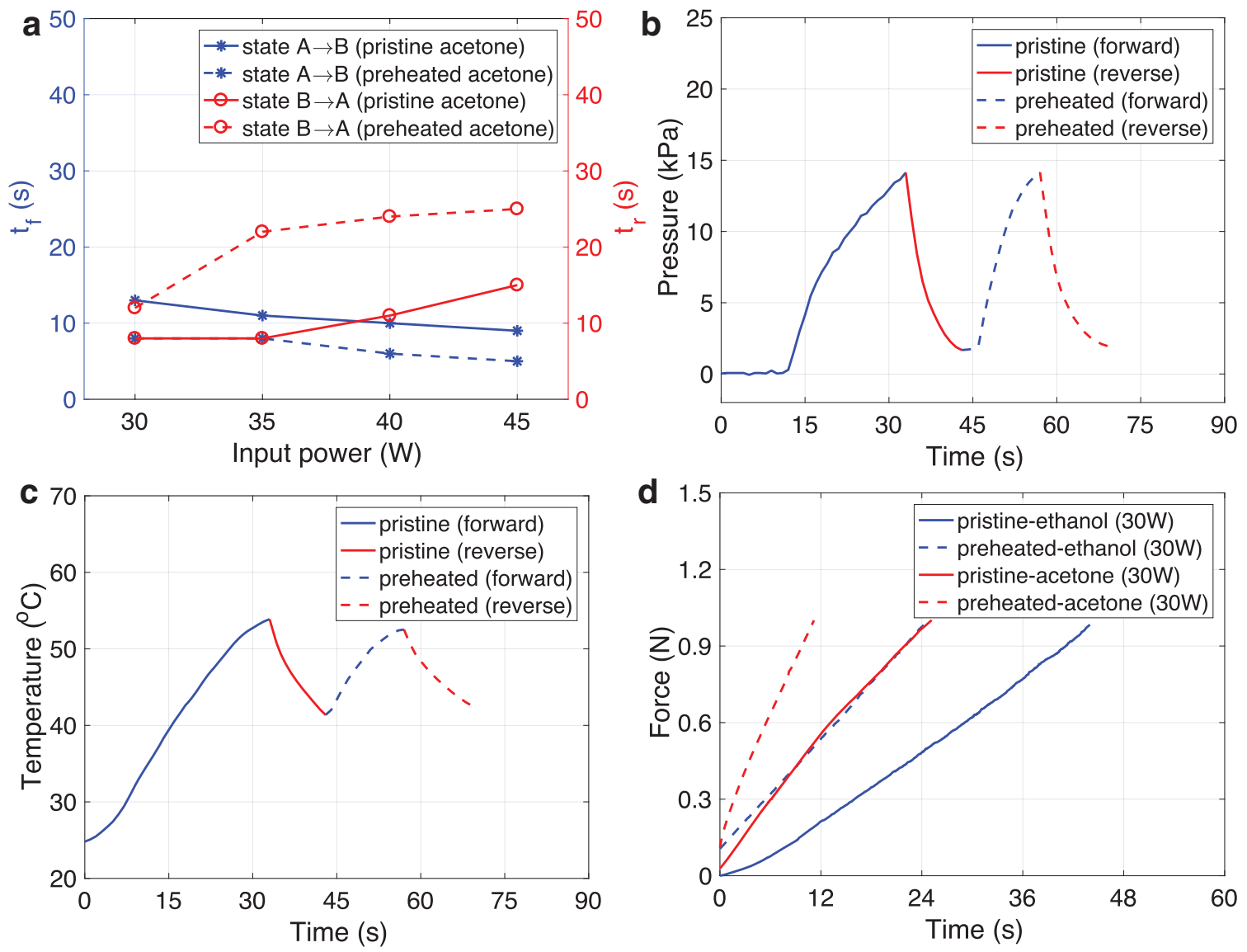

FIG. 7. Acetone as working fluid: response time of the actuator during (a) forward and reverse motions using acetone as working fluid. Change of (b) pressure and (c) temperature with respect to time, (d) comparison of time response of force generated at fingertip using two kinds of liquids. Color images are available online.
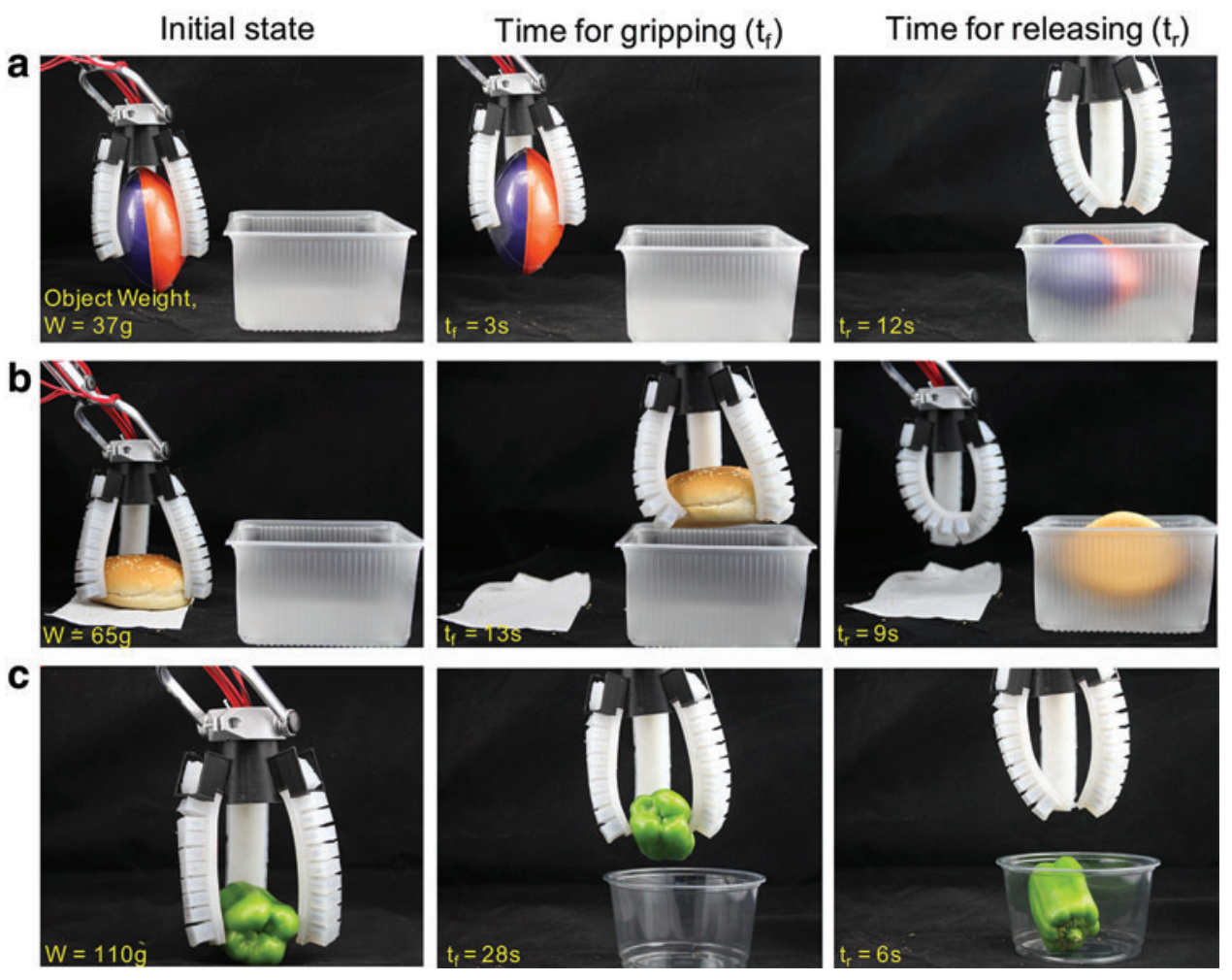

FIG. 8. (a) Applications of electrically operated soft bending actuator handling (a) a toy rugby ball, (b) a soft food item, and (c) a vegetable. Color images are available online.
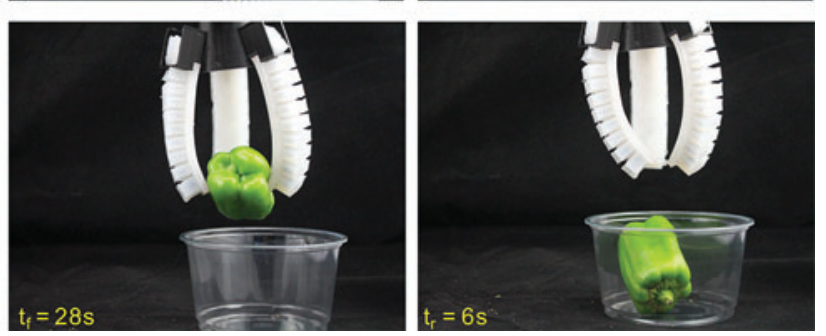
F8 Application of bending motion. In Figure 8, we demonstrate the use of the thermofluidic bending actuator as a soft

AU5 gripper using a DC electrical source. It should be noted that our system performs a similar function to that of the pneumatically operated soft gripper, but using electricity only, and without any motor and compressor/pump. This makes it a compact easy-to-integrate portable system. The greatest advantage of such a compact system is its portability. We connected a three-soft griping finger (based on ethanol) in series, and applied a power ranging from 50 to $64 \mathrm{~W}$ (each finger consumes $<22 \mathrm{~W}$ ), depending on the weight of the object that needs to be gripped and lifted. In Figure 8a-c we showed handling of three soft objects with different weights, that is, handling of a toy rugby ball weighing $37 \mathrm{~g}$ (gripped in $3 \mathrm{~s}$ and released in $12 \mathrm{~s}$ ), soft and delicate bread weighing $65 \mathrm{~g}$ (gripped in $13 \mathrm{~s}$ and released in $9 \mathrm{~s}$ ), and a vegetable weighing $110 \mathrm{~g}$ (gripped in $28 \mathrm{~s}$ and released in $6 \mathrm{~s}$ ). Although all these objects have different shapes, the soft finger adapted its shape accordingly to handle the object in a limited time.

\section{Conclusions}

We have fabricated a soft actuator capable of large motion under a low-voltage electrical actuation. To do so, we used liquid-gaseous phase transition of entrapped fluid inside a predesigned cavity. Such a system could be operated by a low-voltage electric source, making it very compact. We designed and fabricated a porous and flexible heating element from steel microfibers. With the steel wool heating element and ethanol, we could obtain a large bending deformation in $7 \mathrm{~s}$, for a power input of $45 \mathrm{~W}$. This speed of actuation is close to that obtained for pneumatic actuators. As we relied on passive cooling for reversibility, we opted for a corrugated structure with a large surface area that ensured a fast reversibility (in most case within $30 \mathrm{~s}$ ). In addition, we showed that it is possible to improve the response time of the sample by selecting a liquid with lower total heat. Further studies will be conducted to investigate whether the response of the sample can be tuned by selecting a liquid with a suitable boiling point. Finally, we presented a practical application involving operating electrically a bending actuator, for gripping. The proposed technological solution is very compact and easy to integrate in an electrically powered system; it is also very cost-effective, in comparison with most existing actuators.

\section{Experimental Section}

\section{Fabrication of the soft bending actuator}

The soft bending actuator is made of four main parts (as shown in Fig. 2a): (1) a top silicon rubber layer with 11 interconnected chambers, (2) a heating element made of stainless steel wool (Grade-00000) passing through the chambers, (3) an in-extensible bottom layer (cotton fabric), and (4) a bottom layer (silicon rubber) for sealing. Two different molds are 3D-printed to fabricate the soft top and bottom layers, respectively. Well-mixed degassed Ecoflex-50 is poured into the mold for the top layer, and cured at $50^{\circ} \mathrm{C}$ for at least $3 \mathrm{~h}$. We then poured Ecoflex-50 into another mold for the bottom layer, inserted a thin cotton fabric (strain limiting layer that was already wetted with Ecoflex-50) into the mold, and cured the material (the mold was only filled halfway). Heating element (yarn) for the actuator $(1 \times d=400 \times 2 \mathrm{~mm}$, resistance
$\mathrm{R} \cong 20 \Omega$ ) was made by twisting and compacting stainless steel microfibers. It was then connected to copper wires for electrical connection and suitably inserted into the cavities of the top layer. Actuation liquid is poured into cavities through a shutoff valve (Supplementary Fig. S2d) connected to one cavity of top layer. To make final assembly, we poured Ecoflex to fully fill the remaining half of the mold of the bottom layer and laid the top layer (with heating element and valve) over the mold of the bottom layer (Fig. 2b) and cured in the oven. The average size of the sample after fabrication is $1 \times$ $\mathrm{w} \times \mathrm{h}=110 \times 20 \times 20 \mathrm{~mm}$ and the average weight is $\sim 42 \mathrm{~g}$, that can be considered as relatively big soft bending actuator. Once the Ecoflex was all cured, liquid $(2.5 \mathrm{~mL}$ of ethanol or acetone) was pumped into the cavities through the valve, and the valve was fully closed during testing.

\section{Mechanical testing}

The compression test was performed on a steel wool yarn of dimensions $\mathrm{L} \times \mathrm{d}=100 \times 2 \mathrm{~mm}$, using an INSTRON 5944 machine (with a load cell of $2 \mathrm{kN}$ ). First, two holders (a male part with rib of dimension $\mathrm{L} \times \mathrm{b} \times \mathrm{h}=100 \times 1.6 \times 2 \mathrm{~mm}$ and female part with a channel $1 \times \mathrm{d} \times \mathrm{h}=100 \times 2 \times 2 \mathrm{~mm}$ ) (Supplementary Fig. S2e) are 3D-printed to fix on the tensile testing machine. The steel wool yarn is kept in the channel of the female holder and the rib of the male part is allowed to enter the cavity using a load cell of $2 \mathrm{kN}$. The sample was compressed, step by step, to $75 \%$ of its initial diameter (Supplementary Fig. S2f). A two-probe measurement system (Keysight digital multimeter) was used to track the change of resistance with respect to strain. To perform a bending test on a steel wool yarn, a sample $(100 \times 2 \mathrm{~mm})$ was attached on a thick Ecoflex sheet of size $110 \times 10 \mathrm{~mm}$. The ends of the sheet were then affixed on the tensile testing machine. Then, $45 \mathrm{~mm}$ of compression extension (crosshead displacement) was applied on the sheet to ensure a sufficient bending of the sheet and sample (an angle of $>180^{\circ}$ ). A two-probe measurement system (Keysight digital multimeter) was used to track the change of resistance, with respect to the bending strain.

\section{Actuation}

The sample was actuated using a DC voltage source (KORAD DC digital control power supply $60 \mathrm{~V}, 3 \mathrm{~A}$ ) and the deformation was captured using a digital camera (Canon). The trajectory was determined with the help of a graph paper background and a WebPlotDigitizer software (Supplemen- 4 SF3 tary Fig. S3a, b).

The force generated by the fingertip was measured using a $5 \mathrm{~N}$ load sensor in an INSTRON 5944 machine (Supplementary Fig. S3c). First, a suitable casing was 3D-printed to constrain the motion of the finger in all directions except at the fingertip. Another flat rectangular surface was 3D-printed and attached to the load sensor. Once the finger was electrically actuated, the tip was allowed to apply force on the sensor through the flat surface; the force generated was then monitored using a computer.

\section{Other measurements}

The temperature in this experiment was measured using a $\mathrm{K}$-type thermocouple and a thermocouple data logger (pico 
technology). The pressure inside the channel was measured using a digital manometer (KELLER LEO 3, 0-3 bar). A

AU6 K-114 interface converter was used to convert a USB signal to a serial RS485 half-duplex signal, for connection to the computer with a USB port. And the results were monitored using a K-114 Config software. All thermal images were captured using a thermal camera (FLIR A3500SC). The SEM images were obtained using a Zeiss Merlin SEM tool.

\section{EQ1 Data Availability}

The data sets generated during and/or analyzed in this study are available from the corresponding author, upon reasonable request.

\section{Author Disclosure Statement}

The authors declare no financial or nonfinancial competing interest.

\section{Funding Information}

The research reported in this publication was supported by King Abdullah University of Science and Technology, under award number BAS/1/1315-01-01.

\section{Supplementary Material}

Supplementary Data

Supplementary Figure S1

Supplementary Figure S2

Supplementary Figure S3

Supplementary Movie S1

Supplementary Movie S2

Supplementary Movie S3

Supplementary Movie S4

Supplementary Movie S5

Supplementary Movie S6

\section{References}

1. Rus D, Tolley MT. Design, fabrication and control of soft robots. Nature 2015;521:467-475.

2. Laschi C, Mazzolai B, Cianchetti M. Soft robotics: technologies and systems pushing the boundaries of robot abilities. Sci Robot 2016;1:eaah3690.

3. Whitesides GM. Soft robotics. Angew Chem Int Ed 2018; 57:4258-4273.

4. Hines L, Petersen K, Lum GZ, et al. Soft actuators for small-scale robotics. Adv Mater 2017;29:1603483.

5. Ilievski F, Mazzeo AD, Shepherd RF, et al. Soft robotics for chemists. Angew Chem Int Ed 2011;50:1890-1895.

6. Tolley MT, Shepherd RF, Mosadegh B, et al. A resilient, untethered soft robot. Soft Robot 2014;1:213-223.

7. Marchese AD, Katzschmann RK, Rus D. Whole arm planning for a soft and highly compliant $2 \mathrm{D}$ robotic manipulator. IEEE/RSJ International Conference on Intelligent Robots and Systems, Chicago, 2014:554-560.

8. Rafsanjani A, Zhang Y, Liu B, et al. Kirigami skins make a simple soft actuator crawl. Sci Robot 2018;3:eaar7555.

9. Gu G, Zou J, Zhao R, et al. Soft wall-climbing robots. Sci Robot 2018;3:eaat2874.

10. Verma MS, Ainla A, Yang D, et al. A soft tube-climbing robot. Soft Robot 2018;5:133-137.
11. Huichan Z, Kevin O, Shuo L, et al. Optoelectronically innervated soft prosthetic hand via stretchable optical waveguides. Sci Robot 2016;1:eaai7529.

12. Polygerinos P, Correll N, Morin SA, et al. Soft robotics: review of Fluid-Driven intrinsically soft devices; manufacturing, sensing, control, and applications in human-robot interaction. Adv Eng Mater 2017;19:1700016.

13. Hu W, Lum GZ, Mastrangeli M, et al. Small-scale softbodied robot with multimodal Locomotion. Nature 2018; 554:81-85.

14. Do TN, Phan H, Nguyen TQ, et al. Miniature soft electromagnetic actuators for robotic applications. Adv Funct Mater 2018;28:1800244.

15. Boyvat M, Vogt DM, Wood RJ. Ultrastrong and highstroke wireless soft actuators through liquid-gas phase change. Adv Mater Technol 2019;4:1800381.

16. Mirfakhrai T, Madden JDW, Baughman RH. Polymer artificial muscles. Mater Today 2007;10:30-38.

17. Shintake J, Cacucciolo V, Shea H, et al. Soft biomimetic fish robot made of dielectric elastomer actuators. Soft Robot 2018;5:466-474.

18. Behl M, Razzaq MY, Lendlein A. Multifunctional shapememory polymers. Adv Mater 2010;22:3388-3410.

19. Yang Y, Terentjev EM, Wei Y, et al. Solvent-assisted programming of flat polymer sheets into reconfigurable and self-healing 3D structures. Nat Commun 2018;9: 1906.

20. Jin B, Song H, Jiang R, et al. Programming a crystalline shape memory polymer network with thermo- and photoreversible bonds toward a single-component soft robot. Sci Adv 2018;4:eaao3865.

21. Wu J, Yuan C, Ding Z, et al. Multi-shape active composites by $3 \mathrm{D}$ printing of digital shape memory polymers. Sci Rep 2016;6:24224.

22. Han J, Jiang W, Niu D, et al. Untethered soft actuators by liquid-vapor phase transition: remote and programmable actuation. Adv Intell Syst 2019;1:1900109.

23. Nakahara K, Narumi K, Niiyama R, et al. Electric phasechange actuator with inkjet printed flexible circuit for printable and integrated robot prototyping. IEEE International Conference on Robotics and Automation (ICRA), Singapore, 2017:1856-1863.

24. An S, Kang DJ, Yarin AL. A blister-like soft nano-textured thermo-pneumatic actuator as an artificial muscle. Nanoscale 2018;10:16591-16600.

25. Kang DJ, An S, Yarin AL, et al. Programmable soft robotics based on nano-textured thermo-responsive actuators. Nanoscale 2019;11:2065-2070.

26. Zhiwei Z, Qingwei L, Luzhuo C, et al. A large-deformation phase transition electrothermal actuator based on carbon nanotube-elastomer composites. J Mater Chem 2016;4: $1228-1234$.

27. Roland A, Reinhard S, Rainer K, et al. Large area expansion of a soft dielectric membrane triggered by a liquid gaseous phase change. Appl Phys A 2011;105:1-3.

28. Aslan M, Kenneth S, Hod L. Soft material for soft actuators. Nat Commun 2017;8:596.

29. Volkov AG, Foster JC, Baker KD, et al. Mechanical and electrical anisotropy in Mimosa pudica pulvini. Plant Signal Behav 2010;5:1211-1221.

30. Alexander GV, Tejumade A, Emil J. Closing of venus flytrap by electrical stimulation of motor cells. Plant Signal Behav 2007;2:139-145. 
31. Volkov AG, Foster JC, Ashby TA, et al. Mimosa pudica: electrical and mechanical stimulation of plant movement. Plant Cell Environ 2010;33:163-173.

AU7 32. Hill BS, Findlay GP. The power of movement in plants: the role of osmotic machines. Q Rev Biophys 1981;14:173-222.

33. Pioro IL, Rohsenow W, Doerffer S. Nucleate pool-boiling heat transfer. I: review of parametric effects of boiling surface. Int J Heat Mass Transfer 2004;47:5033-5044.

AU8 34. Yu Y, Song G, Sun L. Determinant role of tunneling resistance in electrical conductivity of polymer composites reinforced by well dispersed carbon nanotubes. J Appl Phys 2010;108:084319.

35. Bao WS, Meguid SA, Zhu ZH, et al. Tunneling resistance and its effect on the electrical conductivity of carbon nanotube nanocomposites. J Appl Phys 2012;111:093726.

36. Hong $\mathrm{S}$, Lee $\mathrm{H}$, Lee $\mathrm{J}$, et al. Highly stretchable and transparent metal nanowire heater for wearable electronics applications. Adv Mater 2015;27:4744-4751.
37. Lee Y, Le VT, Kim JG, et al. Versatile, high-power, flexible, stretchable carbon nanotube sheet heating elements tolerant to mechanical damage and severe deformation. Adv Funct Mater 2018;28:1706007.

38. Mosadegh B, Polygerinos P, Keplinger C, et al. Pneumatic networks for soft robotics that actuate rapidly. Adv Funct Mater 2014;24:2163-2170.

Address correspondence to: Gilles Lubineau COHMAS Laboratory

Division of Physical Sciences and Engineering King Abdullah University of Science and Technology Thuwal 23955-6900 Saudi Arabia

E-mail: gilles.lubineau@kaust.edu.sa 


\section{Supplementary Data}

\section{Calculation of Size of the Heating Element}

When current $I$ is passing through a steel wool yarn of resistance $R$, the heat generated $\left(Q_{g}\right)$ per second is equal to the input power, that is, $Q_{g}=P=I^{2} R$. If $\rho$ is the sample resistivity per unit length, then the length of heating element required to achieve the resistance $R$ to generate $Q_{g}$ amount of heat is $L=R / \rho$.

\section{Properties of ethanol}

Density of ethanol at room temperature $\left(T_{0}=25^{\circ} \mathrm{C}\right)$, $D=0.785 \mathrm{~g} / \mathrm{cm}^{3}$

Volume of ethanol in the cavity, $V=2.5 \mathrm{~mL}=2.5 \mathrm{~cm}^{3}$

Total weight of ethanol, $m_{f}=D \times V=1.96 \mathrm{~g}=0.00196 \mathrm{~kg}$

Boiling point of ethanol at $\sim 15 \mathrm{kPa}, T_{1}=82^{\circ} \mathrm{C}$

Corresponding heat capacity, $C=3 \mathrm{~kJ} / \mathrm{kg}$. $K$ and the approximate heat of evaporation, $H=846 \mathrm{~kJ} / \mathrm{kg}$.

\section{Heat required for phase transition}

In this simple approach, we assume the loss of heat to the solid body and surroundings is negligible in pristine condition, then the total heat required for phase transition of liquid is given as

$$
\begin{aligned}
Q_{p} & =m_{f}(C \Delta T+H)=0.00196 \times(3 \times 57+846) \\
& =1.993 \mathrm{~kJ}=1993 \mathrm{~J} .
\end{aligned}
$$

\section{Input power required}

To achieve $1993 \mathrm{~J}$ of energy in $<40 \mathrm{~s}$, the power required is $p=50 \mathrm{~W}$. If the maximum voltage is limited to $\sim 30 \mathrm{~V}$, then the required resistance is $R=V^{2} / p=900 / 50=18 \Omega$.

\section{Size of heating element}

For $2 \mathrm{~mm}$ thick steel wool yarn of Grade-00000, the approximate resistivity per unit length is $\rho=0.5 \Omega / \mathrm{cm}$ (Note: this value may vary slightly depend on the grade of steel wool, the number density of microfiber in the cross-sectional area, and percolation). Then the needed length of the heating element is $\mathrm{L}=\mathrm{R} / \rho=18 / 0.5=36 \mathrm{~mm}$.

For our experiment, we fabricated $40 \mathrm{~mm}$ long steel wool yarn, which results in an approximate resistance of $20 \Omega$.

\section{Discussion on Enhancement of Passive Cooling}

The major cooling enhancement in our selected corrugated shape can come from reduced thermal resistance of the soft body described by following formula: the total thermal resistance of the soft structure is $\mathrm{R}_{\text {total }}=\mathrm{R}_{\text {cond }}+$ $\mathbf{R}_{\text {conv }}+\mathbf{R}_{\text {rad }}$, which depends on the structure of the soft body. A corrugated chamber-like structure has following advantages over a simple rectangular structure (Supplementary Fig. S1a-d): (1) Chamber walls consist of a very thin membrane, which is useful to improve the conduction heat transfer $\left(\mathrm{R}_{\mathrm{cond}}=\frac{x}{K A}\right)$, (2) surface area is very large (Supplementary Fig. S1d), which further improves the conduction, convection $\left(\mathrm{R}_{\mathrm{conv}}=\frac{1}{h_{\text {conv } \mathrm{A}}}\right)$, and radiation $\left(\mathrm{R}_{\text {rad }}=\frac{1}{h_{\text {rad } A} A}\right)$ heat transfer. In addition, the large expansion of chamber walls (bulged state) during actuation reduces the thickness (from $x \rightarrow x_{1}$ ) and increases the area (from $A \rightarrow A_{1}$ ) further, as shown in Supplementary Figure S1a and b. The thermal resistance of the chamber wall also reduces, which can further improve the heat transfer. In other words, we can say that the proposed structure has a programmable thermal resistance; it reduces in actuated state and regains the original value in reverse actuation. The thermal image of a chamber is shown in Supplementary Figure S1c. It shows that the temperature of chamber walls is very high compared with the rest of the actuator, which supports the statements made earlier. That is, the chamber walls have a low thermal resistance due to reduced thickness. Therefore, chamber walls can play a significant role in heat removal from the actuator during reverse actuation. 


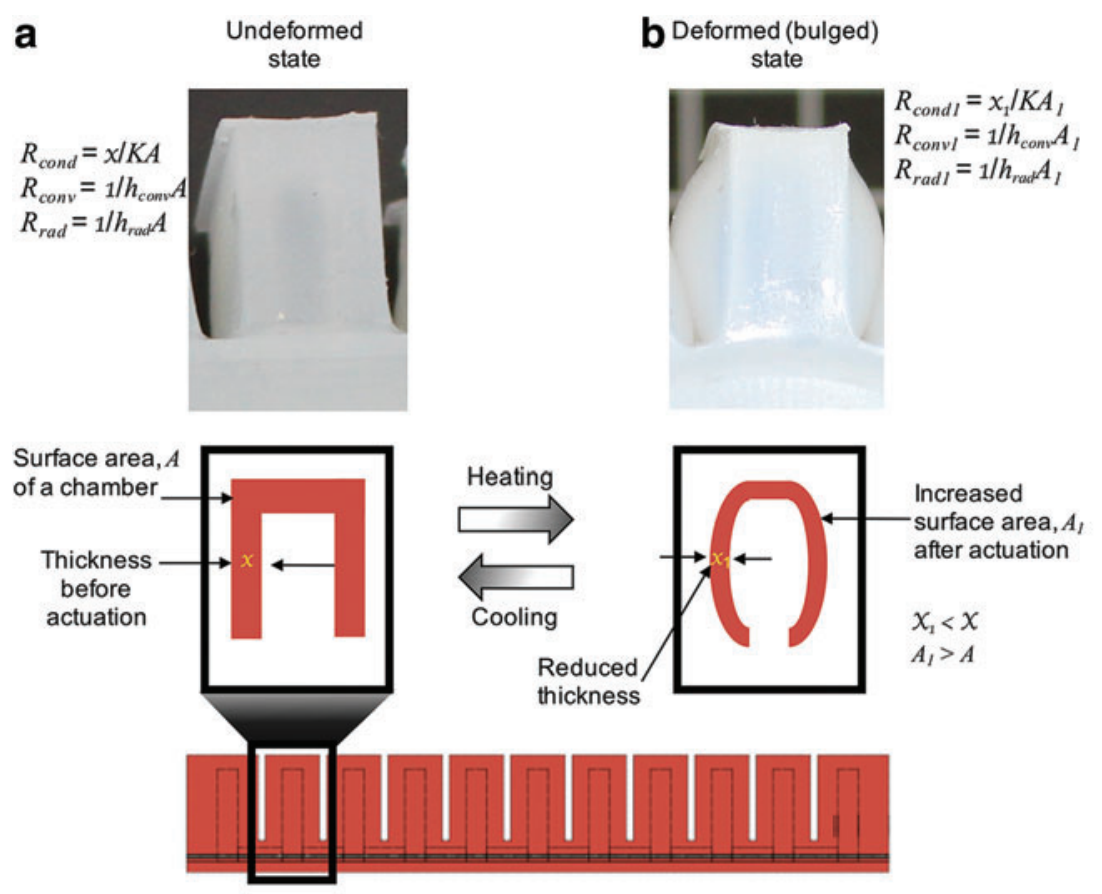

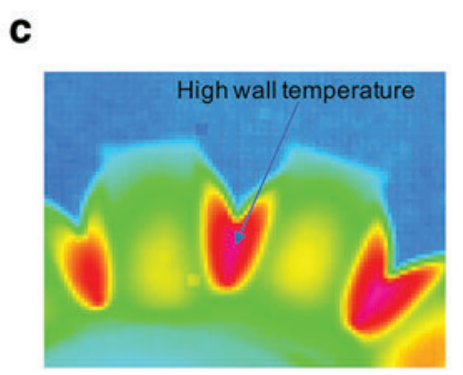

High temperature of chamber walls, implies the low thermal resistance of the wall

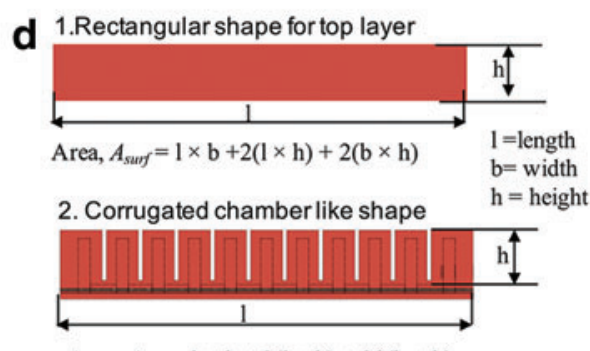

Area, $A_{\text {surf }}=1 \times \mathrm{b}+2(\mathrm{l} \times \mathrm{h})+20(\mathrm{~b} \times \mathrm{h})$

SUPPLEMENTARY FIG. S1. The thermal resistance of the actuator: the (a) undeformed and (b) deformed configuration of a chamber of the actuator and corresponding change in thermal resistance. (c)The thermal image of a chamber showing high wall temperature due to reduced thickness. (d) A simple comparison of the area of a rectangular shape and corrugated shape for the top layer of bending actuator.

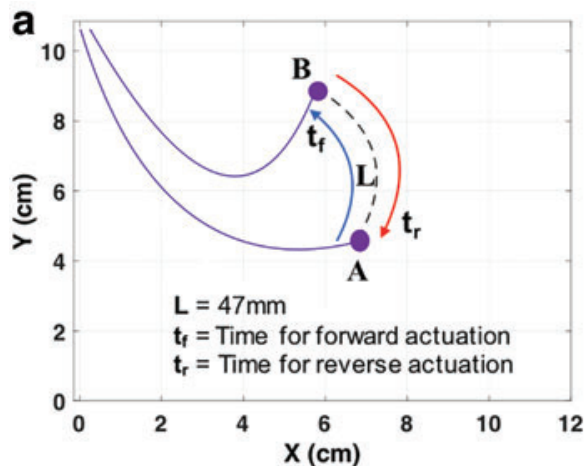

e

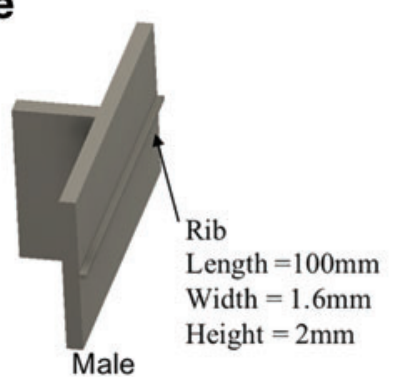

b

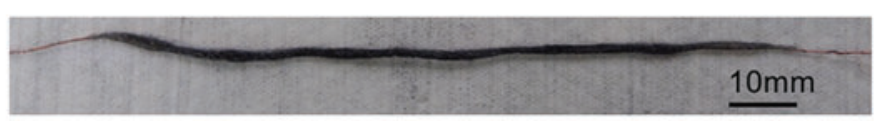

d

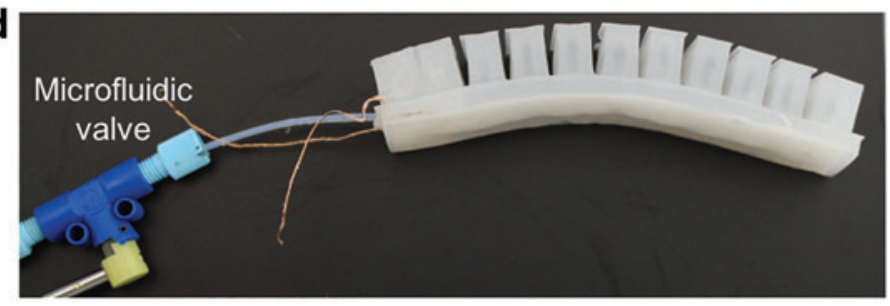

f

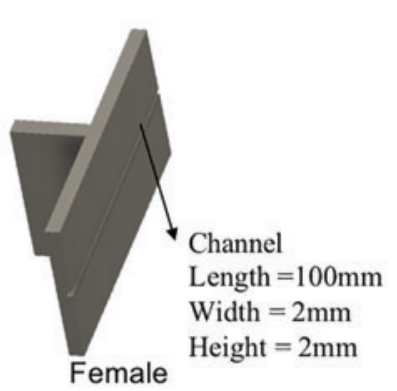

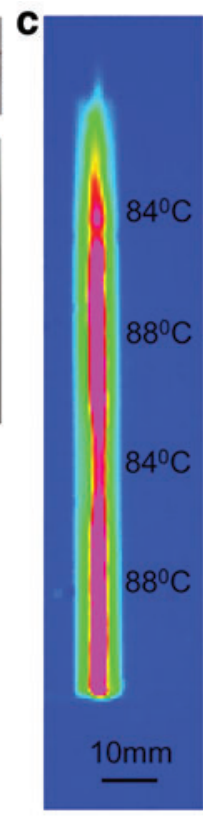

Before compression After compression

SUPPLEMENTARY FIG. S2. Experimental setup 1: (a) Definition of different states (initial state A and final state B) to define the time of actuation of the actuator. (b) The sample heating element used for mechanical testing and thermal analysis. (c) Thermal image of heating element showing uniform heating under $5 \mathrm{~W}$ input power. (d) The final assembling of the soft bending actuator along with microfluidic shutoff valve to supply fluid to the cavity. (e) CAD design for male and female parts used for the compression test of the heating element. (f) The optical camera image of the compression test. 

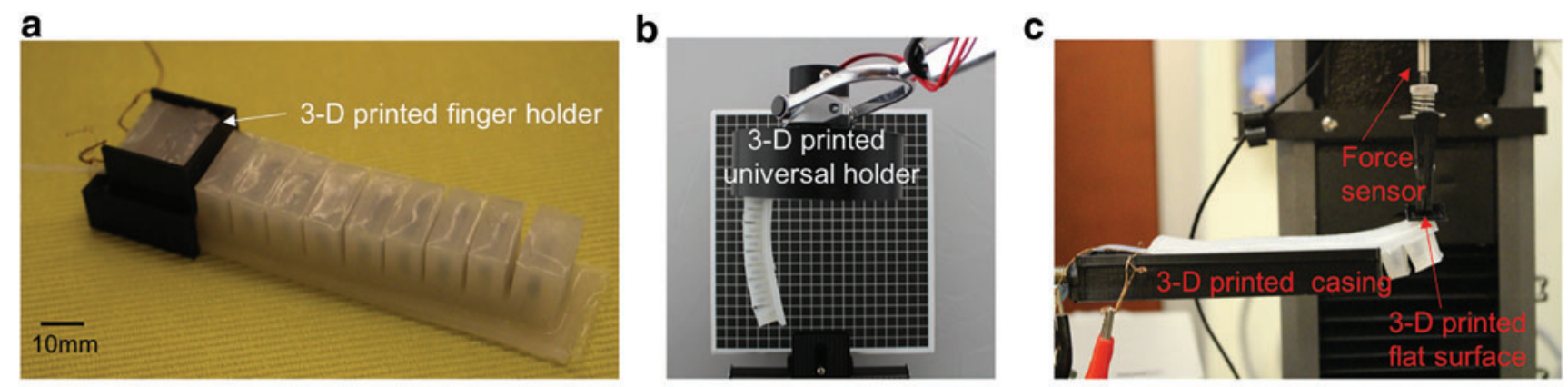

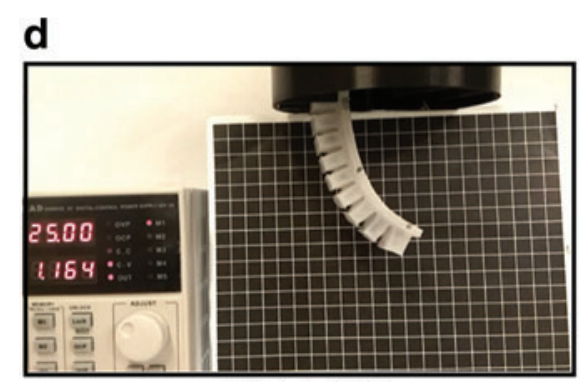

$25 \mathrm{~V}, 1.164 \mathrm{~A}$

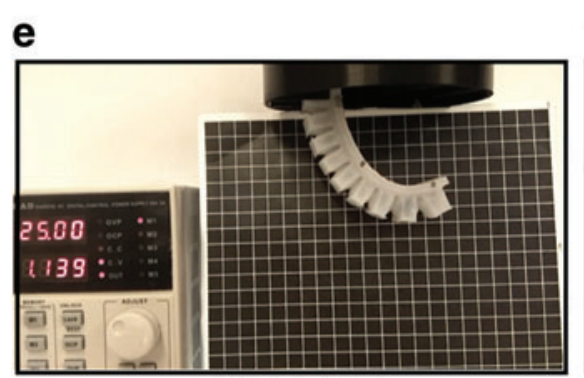

$25 \mathrm{~V}, 1.139 \mathrm{~A}$

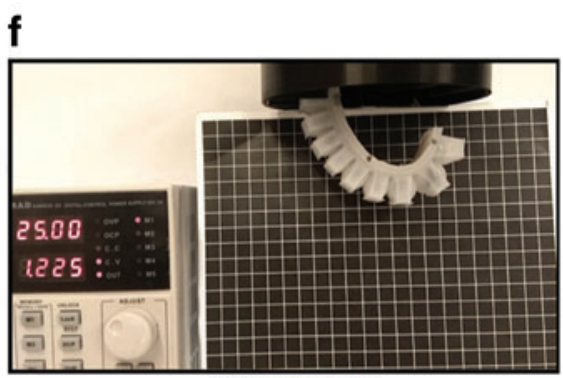

$25 \mathrm{~V}, 1.225 \mathrm{~A}$

SUPPLEMENTARY FIG. S3. Experimental setup 2: (a) 3D-printed small holder to attach the finger with universal holder. (b) Experimental setup for monitoring the bending actuation with graph paper background to capture the trajectory. (c) Experimental setup for measuring the force at the actuator tip. Operating at low voltage and constant power (30 W): (d) initial state, (e) intermediate state, and (f) final state of soft bending actuator in forward direction showing that there is only negligible change in current (and resistance of steel yarn) for a constant input voltage, indicates that the system can operate at constant power without any change in resistance during the bending deformation. 3D, three-dimensional.

SUPPLEMENTARY MOVIE SM1. Comparison between 30 and $45 \mathrm{~W}$ in forward actuation using ethanol.

SUPPLEMENTARY MOVIE SM2. Comparison between ethanol and acetone in forward actuation.

SUPPLEMENTARY MOVIE SM3. Comparison between ethanol and acetone in reverse actuation.

SUPPLEMENTARY MOVIE SM4. Handling of vegetable.

SUPPLEMENTARY MOVIE SM5. Handling of bun.

SUPPLEMENTARY MOVIE SM6. Handling of rugby ball. 


\section{AUTHOR QUERY FOR SORO-2019-0150-VER9-CHELLATTOAN_1P}

AU1: Please identify (highlight or circle) all authors' surnames for accurate indexing citations.

AU2: Please check the correctness of authors' affiliation.

AU3: Citations for Supplementary Figures "S1-S3" are not in sequence. Please check.

AU4: Please note that "Figure" has been changed to "Figure 3" in the sentence, "Figure 3 shows that the time required to reach the $\mathrm{Tb}$ of ethanol can be suitably adjusted by increasing the input power (which depend on cavity size of soft actuator and fluid quantity)."

AU5: Please define "DC."

AU6: Please define "USB."

AU7: Refs. "32-38" have been renumbered to maintain sequential order of citations in the text. Please check.

AU8: Please cite Refs. "34 and 35" in the text.

AU9: Please confirm the corresponding author's name and address.

AU10: Please expand "CAD."

AU11: Please cite Supplementary Movies "SM1 to SM6" in the text.

\section{EDITOR QUERY FOR SORO-2019-0150-VER9-CHELLATTOAN_1F}

EQ1: We have retained "Data Availability" section as it is. Please confirm and check the placement. 